\title{
EATING BEHAVIOURS, FREQUENCY OF CONSUMPTION OF SELECTED FOOD PRODUCTS, AND SELECTED ELEMENTS OF LIFESTYLE AMONG YOUNG DANCERS
}

\author{
Elżbieta Szczepańskal, Barbara Janota ${ }^{1}$, Marika Wlazłol, Martyna Czapla ${ }^{l}$ \\ ${ }^{1}$ Department of Human Nutrition, School of Health Sciences in Bytom, \\ Medical University of Silesia in Katowice, Poland
}

\begin{abstract}
Background. Proper nutrition and balanced physical activity have a beneficial effect on the growth and development of young athletes, supporting their endurance and health, which is particularly important in the period of adolescence.

Objective. Aim of this study was to assess eating behaviours, frequency of consumption of some food products and selected elements of lifestyle among young dancers, as well as to compare the age of the dancers and the assessed behavior. Material and methods. The study included 198 dancers aged 10-15 years. An originally developed questionnaire was used as a research tool.

Results. The largest group of dancers (43\%) spent on training up to 2 hours per week. The recommended 4-5 meals/day were consumed by $61.1 \%$ of dancers, with $87.4 \%$ of respondents having breakfast on a daily basis. The recommendations on the daily intake of several portions of fruit and vegetables were followed by $49 \%$ and $36.9 \%$ of dancers, respectively. It is observed, that the consumption rates of sweets increased, while the consumption rates of fruit decreased with dancers' age. The consumption rates of packed lunch and afternoon snack increased, whereas the consumption rates of dinner decreased with an increasing number of hours spent training.

Conclusions. Eating behaviours and the frequency of consumption of food products by young dancers can be described as varied. Their diet is characterised by regular consumption of meals and a correct frequency of consumption of most food products, including high intake of fruit and vegetables, yoghurt and kefir, as well as low consumption of fast food products. The selected elements of dancers' lifestyle, such as sleep time and physical activity, are mostly correct.
\end{abstract}

Key words: lifestyle, nutrition, dancers

\section{STRESZCZENIE}

Wstęp. Prawidłowe odżywianie i zrównoważona aktywność fizyczna wpływają korzystnie na wzrost i rozwój młodych sportowców, wspierając ich wytrzymałość i kondycję zdrowotną, co jest szczególnie istotne w okresie dojrzewania.

Cel. Celem badań była ocena zachowań żywieniowych, częstości spożycia wybranych produktów spożywczych oraz wybranych elementów stylu życia młodych tancerzy, a także porównanie zależności pomiędzy wiekiem tancerzy a ocenianymi zachowaniami.

Material i metody. Badania przeprowadzono wśród 198 tancerzy w wieku 10-15 lat. Narzędziem badawczym był autorski kwestionariusz ankiety.

Wyniki. Najliczniejsza grupa tancerzy (43\%) przeznaczała na trening do 2 godzin tygodniowo. Zalecane 4-5 posiłków dziennie spożywało 61,1\% tancerzy, śniadanie spożywało codziennie 87,4\% z nich. Zalecenia kilkukrotnego spożywania owoców i warzyw a ciągu dnia przestrzega odpowiednio 49\% i 36,9\% badanych tancerzy. Obserwuje się, że wraz wiekiem tancerzy wzrasta częstość spożycia słodyczy, natomiast maleje częstość spożycia owoców oraz, że wraz z większą ilością godzin spędzanych przez tancerzy na treningu wzrasta częstość spożycia II śniadania i podwieczorku, natomiast maleje częstość spożycia obiadu.

Wnioski. Zachowania żywieniowe oraz częstość spożycia produktów spożywczych przez młodych tancerzy można ocenić jako zróżnicowane. Ich sposób żywienia charakteryzuje się regularnością spożywania poszczególnych posiłków oraz prawidłową częstością spożywania większości produktów spożywczych, w tym wysokim spożyciem owoców i warzyw, jogurtów, kefirów oraz niskim spożyciem produktów typu fast food. Wybrane elementy stylu życia, takie jak czas przeznaczony na sen oraz uprawianie aktywności fizycznej przez młodych tancerzy w większości są właściwe.

Słowa kluczowe: styl życia, odżywianie, tancerze

Corresponding author: Elżbieta Szczepańska, Ph.D, Department of Human Nutrition, School of Health Sciences in Bytom, Medical University of Silesia, Jordana 19 Street, 41-808 Zabrze, Poland, phone: +48 32 275-51-97, e-mail: eszczepanska@sum.edu.pl

(C) Copyright by the National Institute of Public Health - National Institute of Hygiene 


\section{INTRODUCTION}

Providing the right amount of food products that contain the nutrients the body needs, is an important part of the lifestyle of physically active people [1]. Despite the widely available knowledge about proper nutrition, some researchers point to improper eating behavior, which they often equate with a low level of knowledge of the studied group or the lack of reliable nutritional counseling [26].

Proper nutrition and balanced physical activity have a beneficial effect on the body, supporting endurance and health, which are particularly important in the period of adolescence $[8,38]$. According to the World Health Organization, children aged $10-15$ years need at least 60 minutes of daily physical activity of any form to maintain proper body shape, as well as physical and mental efficiency [22,34]. Dancing is one of the forms of physical activity, which combines the awareness of body movement with musical sensitivity. As in other activities, also in this case the effort put into training is associated with increased energy expenditure, and thus the need for properly balanced diet. Having 5 meals daily at fixed times of day in between the training sessions, what is recommended by the Food and Nutrition Institute, is beneficial for the absorption of nutrients necessary for body functions [11]. The presence of various groups of products in the diet allows for optimal supply of these ingredients. According to the recommendations of the Pyramid of Healthy Nutrition and Lifestyle for Children and Youth, daily intake of vegetables and fruits, which contain antioxidant polifenoles and prebiotic fibre, is particularly important [27, 37]. In addition to fruit and vegetables, grain products, which also provide essential ingredients for blood formation (iron and folic acid), should be an important source of fibre [19]. Along with prebiotics, a balanced diet should provide probiotic bacteria, which are found, among other things, in yogurt and kefir, which improve digestion and show hypocholesterolemic effects [28]. The seeds of legumes, which are called superfoods due to their high content of vegetable protein, also play an important role in nutrition [31]. Animal meat, fish and eggs should be a source of wholesome protein in the diet [12]. Fish provide essential unsaturated omega-3 fatty acids, which have anti-inflammatory activity when consumed at a recommended daily amount of 250 $\mathrm{mg}$, thus preventing cancer and cardiovascular diseases [29].

A properly composed diet excludes simple sugars and trans fats. Consumption of simple sugars disrupts satiety, which may result in compulsive eating and overweight, which in turn leads to diet-related diseases. Therefore, a diet with limited content of sweets and processed products rich in simple sugars is recommended [9]. Trans fats found in sweets and fast food products have destructive effects on blood vessel endothelial functions, cause lipid disorders, thereby contributing to the development of cardiovascular diseases [10]. A diet rich in products with high nutritional value has positive effects on the growth and development of young athletes, while the consumption of highly processed products may have negative effects on the body, leading to diet-related diseases $[14,16]$.

Young, active people are particularly vulnerable to the consequences of improper nutrition. Monitoring their lifestyle, including eating behavior monitoring, enables early detection of abnormalities [18].

The aim of this study was to assess eating behaviours, the frequency of consumption of selected food products and selected elements of lifestyle among young dancers, as well as to compare the age of the dancers and the assessed behavior.

\section{MATERIALS AND METHODS}

The study was conducted in winter 2020 among 198 attending classes at dance clubs and ballet schools, living in Śląskie, Łódzkie and Świętokrzyskie provinces. There were 194 girls (98\%) and 4 boys (2\%), aged between 10 and 15 years, in the study group. Consent to participate in the study was obtained from the parents.

An originally designed questionnaire including demographics and 17 questions on eating behaviours and physical activity was used as a research tool. The questionnaire was anonymous. The obtained results were collected and analysed using Microsoft Excel 2010 spreadsheet and Statistica 13 software. The results were presented for the total group and separately for the age groups: 10-12 years and 1315 years. The gamma coefficient was used to assess the relationships between consumption rates for selected products, meals and age, as well as between consumption rates for selected meals and the number of training hours per week. A p-value (p) $<0.05$ was considered statistically significant for all analyses.

\section{RESULTS}

The selected lifestyle elements are presented in Tables 1-3.

The largest group of dancers (43\%) spent on training up to 2 hours per week, as indicated mainly by 10-12-year-olds. Older dancers usually spend 2-3 hours training (Table 1). Swimming was the most common additional physical activity practised by the dancers, and it was more common among 10-12-yearolds than $13-15$-year-olds ( $22.7 \%$ vs. $19 \%)$. Only $16.7 \%$ of dancers engaged in no additional physical activity (Table 2). A total of $61 \%$ of dancers, including more 10 -12-year-olds than $13-15$-year-olds ( $71 \%$ vs. $44 \%)$ 
get the recommended number of sleep hours per day (Table 3).

Selected eating behaviours are shown in Tables 4-10.

The recommended 4-5 meals/day were consumed by $61.1 \%$ of dancers, including $69.6 \%$ of older and
$55.5 \%$ of younger respondents (Table 4). Fruit and vegetables were the most frequently consumed snacks among dancers $(63.6 \%)$, and were more common among 10-12-year-olds (70.6\%) than 13-15-year-olds (53.2\%) (Table 5).

Table 1. Time spent on training per week

\begin{tabular}{|l|c|c|c|c|c|c|}
\hline \multirow{2}{*}{ Number of training hours/age } & \multicolumn{2}{c|}{$10-12$ age } & \multicolumn{2}{c|}{$13-15$ age } & \multicolumn{3}{c|}{ Total } \\
\cline { 2 - 8 } & $\mathrm{n}=119$ & $\%$ & $\mathrm{n}=79$ & $\%$ & $\mathrm{n}=198$ & $\%$ \\
\hline Up to 2 hours & 61 & 51.3 & 24 & 30.4 & 85 & 43 \\
\hline 2-3 hours & 31 & 26 & 28 & 35.4 & 59 & 30 \\
\hline 4-5 hours & 15 & 12.6 & 15 & 19 & 30 & 15 \\
\hline Over 5 hours & 12 & 10.1 & 12 & 15.2 & 24 & 12 \\
\hline
\end{tabular}

Table 2. Additional physical activity

\begin{tabular}{|l|c|c|c|c|c|c|}
\hline \multirow{2}{*}{ Additional activity/age } & \multicolumn{2}{c|}{$10-12$ age } & \multicolumn{2}{c|}{$13-15$ age } & \multicolumn{3}{c|}{ Total } \\
\cline { 2 - 7 } & $\mathrm{n}=119$ & $\%$ & $\mathrm{n}=79$ & $\%$ & $\mathrm{n}=198$ & $\%$ \\
\hline Running & 16 & 13.4 & 11 & 14 & 27 & 13.6 \\
\hline Swimming & 27 & 22.7 & 15 & 19 & 42 & 21.2 \\
\hline Cycling & 9 & 7.6 & 10 & 13 & 19 & 9.6 \\
\hline Team sport & 5 & 4.2 & 9 & 11 & 14 & 7.1 \\
\hline Gymnastics & 24 & 20.2 & 10 & 13 & 34 & 17.2 \\
\hline Others, e.g. horse riding, acrobatics & 21 & 17.6 & 8 & 10 & 29 & 14.6 \\
\hline No other physical activities than dancing & 17 & 14.3 & 16 & 20 & 33 & 16.7 \\
\hline
\end{tabular}

Table 3. Number of hours of sleep per day

\begin{tabular}{|c|c|c|c|c|c|c|}
\hline \multirow{2}{*}{ Number of hours of sleep/age } & \multicolumn{2}{|c|}{$10-12$ age } & \multicolumn{2}{|c|}{$13-15$ age } & \multicolumn{2}{c|}{ Total } \\
\cline { 2 - 8 } & $\mathrm{n}=119$ & $\%$ & $\mathrm{n}=79$ & $\%$ & $\mathrm{n}=198$ & 76 \\
\hline Less than 8 hours & 32 & 27 & 44 & 56 & 76 & 61 \\
\hline 8-11 hours & 85 & 71 & 35 & 44 & 120 & 61 \\
\hline Over 11 hours & 2 & 2 & 0 & 0 & 2 & 1 \\
\hline
\end{tabular}

Table 4. Number of meals

\begin{tabular}{|l|c|c|c|c|c|c|}
\hline \multirow{2}{*}{ Number of meals/age } & \multicolumn{2}{|c|}{$10-12$ age } & \multicolumn{2}{c|}{$13-15$ age } & \multicolumn{3}{c|}{ Total } \\
\cline { 2 - 8 } & $\mathrm{n}=119$ & $\%$ & $\mathrm{n}=79$ & $\%$ & $\mathrm{n}=198$ & \multicolumn{2}{c|}{$\%$} \\
\hline 2 or less & 1 & 0.8 & 3 & 3.8 & 4 & 2 \\
\hline 3 & 44 & 37 & 16 & 20.3 & 60 & 30.3 \\
\hline $4-5$ & 66 & 55.5 & 55 & 69.6 & 121 & 61.1 \\
\hline More than 5 & 8 & 6.7 & 5 & 6.3 & 13 & 6.6 \\
\hline
\end{tabular}

Table 5. The most frequently consumed snacks

\begin{tabular}{|l|c|c|c|c|c|c|}
\hline \multirow{2}{*}{ Most frequently eaten snacks/age } & \multicolumn{2}{|c|}{$10-12$ age } & \multicolumn{2}{c|}{$13-15$ age } & \multicolumn{2}{c|}{ Total } \\
\cline { 2 - 8 } & $\mathrm{n}=119$ & $\%$ & $\mathrm{n}=79$ & $\%$ & $\mathrm{n}=198$ & $\%$ \\
\hline Vegetables or fruit & 84 & 70.6 & 42 & 53.2 & 126 & 63.6 \\
\hline Sweets & 10 & 8.4 & 24 & 30.4 & 34 & 17.2 \\
\hline Salty snacks & 12 & 10.1 & 5 & 6.3 & 17 & 8.6 \\
\hline Others, e.g. nuts, yoghurts & 7 & 5.9 & 7 & 8.8 & 14 & 7.1 \\
\hline I do not eat snacks in between meals & 6 & 5 & 1 & 1.3 & 7 & 3.5 \\
\hline
\end{tabular}


Table 6. Frequency of meals

\begin{tabular}{|c|c|c|c|c|c|c|c|c|c|}
\hline & \multirow{2}{*}{ Frequency of meals } & \multicolumn{2}{|c|}{$10-12$ age } & \multicolumn{2}{|c|}{$13-15$ age } & \multicolumn{2}{|c|}{ Total } & \multicolumn{2}{|c|}{$\begin{array}{c}\text { Frequency of } \\
\text { meals/age }\end{array}$} \\
\hline & & $\mathrm{n}=119$ & $\%$ & $\mathrm{n}=79$ & $\%$ & $\mathrm{n}=198$ & $\%$ & gamma & $\mathrm{p}$ \\
\hline \multirow{4}{*}{ 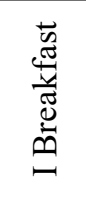 } & Every day & 103 & 86.5 & 70 & 88.6 & 173 & 87.4 & \multirow{4}{*}{0.06} & \multirow{4}{*}{0.59} \\
\hline & Several times a week & 14 & 11.8 & 8 & 10.1 & 22 & 11.1 & & \\
\hline & Several times a month & 0 & 0 & 0 & 0 & 0 & 0 & & \\
\hline & Not consume & 2 & 1.7 & 1 & 1.3 & 3 & 1.5 & & \\
\hline \multirow{4}{*}{ 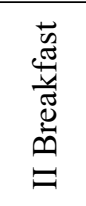 } & \begin{tabular}{|l|} 
Every day \\
\end{tabular} & 54 & 45.4 & 40 & 50.6 & 94 & 47.5 & \multirow{4}{*}{0.16} & \multirow{4}{*}{0.02} \\
\hline & Several times a week & 45 & 37.8 & 30 & 38 & 75 & 37.9 & & \\
\hline & Several times a month & 2 & 1.7 & 3 & 3.8 & 5 & 2.5 & & \\
\hline & Not consume & 18 & 15.1 & 6 & 7.6 & 24 & 12.1 & & \\
\hline \multirow{4}{*}{$\begin{array}{l}\dot{\bar{\Xi}} \\
\dot{\Xi}\end{array}$} & Every day & 114 & 95.8 & 70 & 88.6 & 184 & 93 & \multirow{4}{*}{-0.24} & \multirow{4}{*}{0.08} \\
\hline & Several times a week & 4 & 3.4 & 8 & 10.1 & 12 & 6 & & \\
\hline & Several times a month & 0 & 0 & 0 & 0 & 0 & 0 & & \\
\hline & Not consume & 1 & 0.8 & 1 & 1.3 & 2 & 1 & & \\
\hline \multirow{4}{*}{ 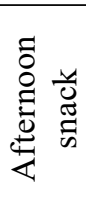 } & Every day & 17 & 14.3 & 15 & 19 & 32 & 16.1 & \multirow{4}{*}{0.21} & \multirow{4}{*}{0.00} \\
\hline & Several times a week & 51 & 42.9 & 34 & 43 & 85 & 43 & & \\
\hline & Several times a month & 15 & 12.6 & 12 & 15.2 & 27 & 13.6 & & \\
\hline & Not consume & 36 & 30.2 & 18 & 22.8 & 54 & 27.3 & & \\
\hline \multirow{4}{*}{$\begin{array}{l}\overline{\bar{D}} \\
\overline{0} \\
\overline{\tilde{n}}\end{array}$} & Every day & 101 & 84.9 & 58 & 73.4 & 159 & 80.3 & \multirow{4}{*}{0.05} & \multirow{4}{*}{0.47} \\
\hline & Several times a week & 14 & 11.7 & 17 & 21.6 & 31 & 15.7 & & \\
\hline & Several times a month & 2 & 1.7 & 2 & 2.5 & 4 & 2 & & \\
\hline & Not consume & 2 & 1.7 & 2 & 2.5 & 4 & 2 & & \\
\hline
\end{tabular}

Breakfast is consumed on a daily basis by $87.4 \%$ of respondents (including $86.5 \%$ of younger and $88.6 \%$ of older dancers), whereas supper by $80.3 \%$ $(84.9 \%$ and $73.4 \%$, respectively) of respondents. Daily consumption of dinner was declared by $93 \%$ of respondents, including $95.8 \%$ of younger and $88.6 \%$ of older dancers. Packed lunch and afternoon snack are not consumed by $12.1 \%$ and $27.3 \%$ of respondents, respectively, as indicated by more younger than older respondents in both cases. The frequency of eating a second breakfast and afternoon snack increases with age. (Table 6).

The recommendations on the daily intake of several portions of fruit and vegetables were followed by $49 \%$ and $36.9 \%$ of dancers, respectively, as indicated by more younger respondents in both cases. The frequency of fruit consumption decreases with age. Also, $36.9 \%$ of dancers, mainly older respondents, consumed vegetables once a day. Legumes are most often consumed at a frequency of several times a month $(46.5 \%)$, as indicated by a higher number of older $(57 \%)$ than younger $(39.5 \%)$ dancers.

Bread was consumed on a daily basis by $92.4 \%$ of respondents, including $91.6 \%$ of younger and $93.6 \%$ of older respondents, whereas rice and groats are consumed several Times a week by $57.6 \%$ of respondents, as indicated by a higher number of younger dancers $(59.7 \%$ vs. $54.4 \%)$.
Fermented milk products, such as yoghurts and kefirs, are consumed several times a week by most respondents, as indicated by $43 \%$, including $42 \%$ of younger and $44.3 \%$ of older dancers.

Fish is eaten several times a month by $55 \%$ of respondents, including $52.1 \%$ of younger and $59.5 \%$ of older dancers. This was the most frequently indicated answer among all dancers. Meat and meat products, such as lunch meats, on the other hand, are usually consumed several times a week, as declared by $44.9 \%$ of respondents $(50.6 \%$ of older and $41.2 \%$ of younger respondents) (Table 7).

The majority of respondents declared that they consume sweets several times weekly (35.9\%), with a similar percentage in both groups (36.1\% of younger and $35.5 \%$ of older dancers). Everyday consumption of sweets was declared by $35.9 \%$ of respondents, including twice daily by $18.7 \%$ of dancers, as indicated mainly by older respondents in both cases. The frequency of consumption of sweets increases with age.

Fast-food products are most often consumed several times a month, as declared by $66.7 \%$ of respondents, with a similar percentage of younger and older dancers (66.4\% and $67.1 \%$, respectively) (Table 8 ).

Tea is most often consumed several times a day by $51.5 \%$ of respondents, including $54.6 \%$ of younger and $46.8 \%$ of older dancers. Milk and milk beverages are mainly consumed several times a week $(40 \%$ of 
Table 7. The frequency of consumption of selected products

\begin{tabular}{|c|c|c|c|c|c|c|c|c|c|}
\hline \multirow{2}{*}{\multicolumn{2}{|c|}{ Frequency of consumption }} & \multicolumn{2}{|c|}{$10-12$ age } & \multicolumn{2}{|c|}{$13-15$ age } & \multicolumn{2}{|c|}{ Total } & \multicolumn{2}{|c|}{$\begin{array}{c}\text { Frequency of } \\
\text { consumption/ age }\end{array}$} \\
\hline & & $\mathrm{n}=119$ & $\%$ & $\mathrm{n}=79$ & $\%$ & $\mathrm{n}=198$ & $\%$ & gamma & $\mathrm{p}$ \\
\hline \multirow{5}{*}{ 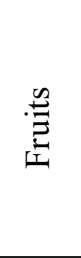 } & Several times a day & 61 & 51.3 & 36 & 45.6 & 97 & 49 & \multirow{5}{*}{-0.16} & \multirow{5}{*}{0.01} \\
\hline & Once a day & 41 & 34.4 & 26 & 32.9 & 67 & 34 & & \\
\hline & Several times a week & 15 & 12.6 & 14 & 17.7 & 29 & 14.5 & & \\
\hline & Several times a month & 2 & 1.7 & 2 & 2.5 & 4 & 2 & & \\
\hline & Not consume & 0 & 0 & 1 & 1.3 & 1 & 0.5 & & \\
\hline \multirow{5}{*}{$\begin{array}{l}\frac{0}{0} \\
\frac{\pi}{0} \\
\stackrel{0}{0} \\
\infty \\
\infty \\
0\end{array}$} & Several times a day & 50 & 42 & 23 & 29.1 & 73 & 36.9 & \multirow{5}{*}{-0.12} & \multirow{5}{*}{0.06} \\
\hline & Once a day & 40 & 33.6 & 33 & 41.8 & 73 & 36.9 & & \\
\hline & Several times a week & 24 & 20.2 & 20 & 25.3 & 44 & 22.2 & & \\
\hline & Several times a month & 4 & 3.4 & 2 & 2.5 & 6 & 3 & & \\
\hline & Not consume & 1 & 0.8 & 1 & 1.3 & 2 & 1 & & \\
\hline \multirow{5}{*}{ 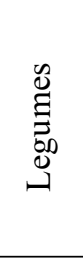 } & Several times a day & 4 & 3.4 & 0 & 0 & 4 & 2 & \multirow{5}{*}{-0.1} & \multirow{5}{*}{0.12} \\
\hline & Once a day & 9 & 7.5 & 4 & 5.1 & 13 & 6.6 & & \\
\hline & Several times a week & 39 & 32.8 & 20 & 25.3 & 59 & 29.8 & & \\
\hline & Several times a month & 47 & 39.5 & 45 & 57 & 92 & 46.5 & & \\
\hline & Not consume & 20 & 16.8 & 10 & 12.6 & 30 & 15.1 & & \\
\hline \multirow{5}{*}{ 芯 } & Several times a day & 70 & 58.8 & 43 & 54.4 & 113 & 57.1 & \multirow{5}{*}{-0.03} & \multirow{5}{*}{0.70} \\
\hline & Once a day & 39 & 32.8 & 31 & 39.2 & 70 & 35.3 & & \\
\hline & Several times a week & 8 & 6.8 & 4 & 5.1 & 12 & 6.1 & & \\
\hline & Several times a month & 1 & 0.8 & 1 & 1.3 & 2 & 1 & & \\
\hline & Not consume & 1 & 0.8 & 0 & 0 & 1 & 0.5 & & \\
\hline \multirow{5}{*}{ 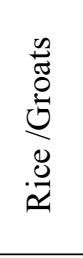 } & Several times a day & 3 & 2.5 & 0 & 0 & 3 & 1.5 & \multirow{5}{*}{-0.13} & \multirow{5}{*}{0.06} \\
\hline & Once a day & 8 & 6.7 & 6 & 7.6 & 14 & 7.1 & & \\
\hline & Several times a week & 71 & 59.7 & 43 & 54.4 & 114 & 57.6 & & \\
\hline & Several times a month & 34 & 28.6 & 27 & 34.2 & 61 & 30.8 & & \\
\hline & Not consume & 3 & 2.5 & 3 & 3.8 & 6 & 3 & & \\
\hline \multirow{5}{*}{ 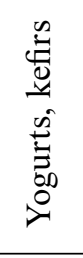 } & Several times a day & 17 & 14.3 & 6 & 7.6 & 23 & 11.6 & \multirow{5}{*}{-0.06} & \multirow{5}{*}{0.32} \\
\hline & Once a day & 26 & 21.8 & 20 & 25.3 & 46 & 23.2 & & \\
\hline & Several times a week & 50 & 42 & 35 & 44.3 & 85 & 43 & & \\
\hline & Several times a month & 19 & 16 & 17 & 21.5 & 36 & 18.2 & & \\
\hline & Not consume & 7 & 5.9 & 1 & 1.3 & 8 & 4 & & \\
\hline & Several times a day & 1 & 0.8 & 0 & 0 & 1 & 0.5 & & \\
\hline & Once a day & 0 & 0 & 1 & 1.3 & 1 & 0.5 & & \\
\hline$\overline{\underline{n}}$ & Several times a week & 39 & 32.8 & 15 & 19 & 54 & 27.3 & 0.00 & 0.98 \\
\hline & Several times a month & 62 & 52.1 & 47 & 59.5 & 109 & 55 & & \\
\hline & Not consume & 17 & 14.3 & 16 & 20.2 & 33 & 16.7 & & \\
\hline & Several times a day & 21 & 17.6 & 9 & 11.4 & 30 & 15.2 & & \\
\hline$\stackrel{\infty}{\tilde{E}}$ & Once a day & 40 & 33.6 & 18 & 22.8 & 58 & 29.3 & & \\
\hline$\overline{0}$ & Several times a week & 49 & 41.2 & 40 & 50.6 & 89 & 44.9 & 0.07 & 0.24 \\
\hline$\sum_{\Sigma}^{\infty}$ & Several times a month & 6 & 5.1 & 8 & 10.1 & 14 & 7.1 & & \\
\hline & Not consume & 3 & 2.5 & 4 & 5.1 & 7 & 3.5 & & \\
\hline
\end{tabular}


Table 8. Frequency of consumption of sweets and fast-food products

\begin{tabular}{|c|c|c|c|c|c|c|c|c|c|}
\hline \multirow{2}{*}{\multicolumn{2}{|c|}{ Frequency of consumption }} & \multicolumn{2}{|c|}{ 10-12 age } & \multicolumn{2}{|c|}{ 13-15 age } & \multicolumn{2}{|c|}{ Total } & \multicolumn{2}{|c|}{$\begin{array}{c}\text { Frequency of } \\
\text { consumption/age }\end{array}$} \\
\hline & & $\mathrm{n}=119$ & $\%$ & $\mathrm{n}=79$ & $\%$ & $\mathrm{n}=198$ & $\%$ & gamma & $\mathrm{p}$ \\
\hline \multirow{5}{*}{ 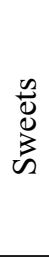 } & Several times a day & 20 & 16.8 & 17 & 21.5 & 37 & 18.7 & \multirow{5}{*}{0.2} & \multirow{5}{*}{0.00} \\
\hline & Once a day & 17 & 14.3 & 17 & 21.5 & 34 & 17.2 & & \\
\hline & Several times a week & 43 & 36.1 & 28 & 35.5 & 71 & 35.9 & & \\
\hline & Several times a month & 34 & 28.6 & 15 & 19 & 49 & 24.7 & & \\
\hline & Not consume & 5 & 4.2 & 2 & 2.5 & 7 & 3.5 & & \\
\hline \multirow{5}{*}{ 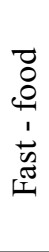 } & Several times a day & 1 & 0.8 & 0 & 0 & 1 & 0.5 & \multirow{5}{*}{0.12} & \multirow{5}{*}{0.12} \\
\hline & Once a day & 2 & 1.7 & 2 & 2.5 & 4 & 2 & & \\
\hline & Several times a week & 7 & 5.9 & 6 & 7.6 & 13 & 6.6 & & \\
\hline & Several times a month & 79 & 66.4 & 53 & 67.1 & 132 & 66.7 & & \\
\hline & Not consume & 30 & 25.2 & 18 & 22.8 & 48 & 24.2 & & \\
\hline
\end{tabular}

Table 9. Frequency of consumption of beverages

\begin{tabular}{|c|c|c|c|c|c|c|c|c|c|}
\hline \multirow{2}{*}{\multicolumn{2}{|c|}{ Frequency of consumption }} & \multicolumn{2}{|c|}{$10-12$ age } & \multicolumn{2}{|c|}{$13-15$ age } & \multicolumn{2}{|c|}{ Total } & \multicolumn{2}{|c|}{$\begin{array}{c}\text { Frequency of } \\
\text { consumption/age }\end{array}$} \\
\hline & & $\mathrm{n}=119$ & $\%$ & $\mathrm{n}=79$ & $\%$ & $\mathrm{n}=198$ & $\%$ & gamma & $\mathrm{p}$ \\
\hline \multirow{5}{*}{$\stackrel{\mathscr{E}}{\oplus}$} & Several times a day & 65 & 54.6 & 37 & 46.8 & 102 & 51.5 & \multirow{5}{*}{-0.13} & \multirow{5}{*}{0.05} \\
\hline & Once a day & 41 & 34.5 & 25 & 31.7 & 66 & 33.3 & & \\
\hline & Several times a week & 12 & 10.1 & 13 & 16.5 & 25 & 12.6 & & \\
\hline & Several times a month & 1 & 0.8 & 2 & 2.5 & 3 & 1.5 & & \\
\hline & Not consume & 0 & 0 & 2 & 2.5 & 2 & 1.1 & & \\
\hline \multirow{5}{*}{ 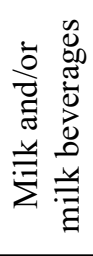 } & Several times a day & 12 & 10.1 & 8 & 10.1 & 20 & 10.1 & \multirow{5}{*}{0.02} & \multirow{5}{*}{0.76} \\
\hline & Once a day & 33 & 27.7 & 22 & 27.8 & 55 & 27.8 & & \\
\hline & Several times a week & 53 & 44.5 & 26 & 33 & 79 & 40 & & \\
\hline & Several times a month & 14 & 11.8 & 17 & 21.5 & 31 & 15.6 & & \\
\hline & Not consume & 7 & 5.9 & 6 & 7.6 & 13 & 6.5 & & \\
\hline \multirow{5}{*}{ 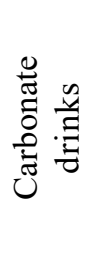 } & Several times a day & 3 & 2.5 & 1 & 1.3 & 4 & 2 & \multirow{5}{*}{0.04} & \multirow{5}{*}{0.47} \\
\hline & Once a day & 3 & 2.5 & 4 & 5.1 & 7 & 3.5 & & \\
\hline & Several times a week & 19 & 16 & 11 & 13.9 & 30 & 15.2 & & \\
\hline & Several times a month & 57 & 47.9 & 42 & 53.2 & 99 & 50 & & \\
\hline & Not consume & 37 & 31.1 & 21 & 26.5 & 58 & 29.3 & & \\
\hline \multirow{5}{*}{ 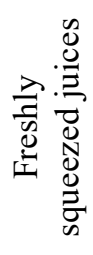 } & Several times a day & 13 & 11 & 2 & 2.5 & 15 & 7.6 & \multirow{5}{*}{-0.10} & \multirow{5}{*}{0.10} \\
\hline & Once a day & 25 & 21 & 13 & 16.5 & 38 & 19.2 & & \\
\hline & Several times a week & 45 & 37.8 & 28 & 35.4 & 73 & 36.9 & & \\
\hline & Several times a month & 30 & 25.2 & 30 & 38 & 60 & 30.3 & & \\
\hline & Not consume & 6 & 5 & 6 & 7.6 & 12 & 6 & & \\
\hline \multirow{5}{*}{ 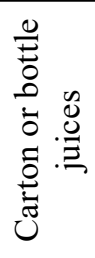 } & Several times a day & 10 & 8.4 & 7 & 8.9 & 17 & 8.6 & \multirow{5}{*}{-0.05} & \multirow{5}{*}{0.42} \\
\hline & Once a day & 13 & 10.9 & 11 & 14 & 24 & 12.1 & & \\
\hline & Several times a week & 33 & 27.7 & 24 & 30.3 & 57 & 28.8 & & \\
\hline & Several times a month & 54 & 45.4 & 27 & 34.2 & 81 & 40.9 & & \\
\hline & Not consume & 9 & 7.6 & 10 & 12.6 & 19 & 9.6 & & \\
\hline
\end{tabular}




\begin{tabular}{|c|c|c|c|c|c|c|c|c|c|}
\hline \multirow{5}{*}{$\begin{array}{l}\tilde{y} \\
\vec{\Xi} \\
\vec{\Xi} \\
\overrightarrow{00} \\
\vec{\Xi} \\
\overrightarrow{\mid I}\end{array}$} & Several times a day & 2 & 1.7 & 0 & 0 & 2 & 1.1 & \multirow{5}{*}{0.11} & \multirow{5}{*}{0.29} \\
\hline & Once a day & 0 & 0 & 1 & 1.3 & 1 & 0.5 & & \\
\hline & Several times a week & 1 & 0.8 & 2 & 2.5 & 3 & 1.5 & & \\
\hline & Several times a month & 5 & 4.2 & 16 & 20.2 & 21 & 10.6 & & \\
\hline & Not consume & 111 & 93.3 & 60 & 76 & 171 & 86.3 & & \\
\hline \multirow{5}{*}{$\frac{\overline{ \pm}}{\vec{\pi}}$} & Several times a day & 109 & 91.6 & 76 & 96.2 & 185 & 93.4 & \multirow{5}{*}{0.11} & \multirow{5}{*}{0.10} \\
\hline & Once a day & 5 & 4.2 & 1 & 1.3 & 6 & 3.1 & & \\
\hline & Several times a week & 5 & 4.2 & 2 & 2.5 & 7 & 3.5 & & \\
\hline & Several times a month & 0 & 0 & 0 & 0 & 0 & 0 & & \\
\hline & Not consume & 0 & 0 & 0 & 0 & 0 & 0 & & \\
\hline
\end{tabular}

respondents), as declared by $44.5 \%$ of younger and $33 \%$ of older respondents. Carbonated drinks, which are not recommended in the diet, are consumed by most adolescents several times a month (50\%), as indicated by fewer younger dancers $(47.9 \%$ vs. $53.2 \%)$.

The majority of respondents $(36.9 \%$, including $37.8 \%$ of younger and $35.4 \%$ of older dancers) consume freshly squeezed juices several times a week. Carton or bottle juices are usually consumed several times a month, as declared by $40.9 \%$ of respondents $(45.4 \%$ of younger and $34.2 \%$ of older dancers).

Energy drinks, which are generally not recommended, are not consumed by $86.3 \%$ of dancers, as indicated by a higher number of younger (93.3\%) than older $(76 \%)$ respondents. The recommended daily consumption of water (several times a day) was declared by $93.4 \%$ of respondents $(91.6 \%$ of younger and $96.2 \%$ of older dancers). There was no correlation between the frequency of consumption of selected drinks and the age of the dancers.(Table 9).

Table 10. Relationship between the number of training hours per week and consumption of meals

\begin{tabular}{|l|c|c|}
\hline \multicolumn{1}{|c|}{ Name of meal } & gamma & $\mathrm{p}$ \\
\hline I breakfast & 0.209 & 0.095 \\
\hline II breakfast & 0.177 & $\mathbf{0 . 0 1 6}$ \\
\hline Dinner & -0.354 & $\mathbf{0 . 0 1 6}$ \\
\hline Afternoon snack & 0.251 & $\mathbf{0 . 0 0 0}$ \\
\hline Supper & 0.097 & 0.283 \\
\hline
\end{tabular}

The frequency of consuming packed breakfast and afternoon snack increased and the frequency of consuming dinner decreased with an increasing number of training hours.

\section{DISCUSSION}

The analysis of our results showed that $61.1 \%$ of dancers have 4-5 meals a day, as recommended by the Food and Nutrition Institute [37]. Similar results were obtained by Bielec and Goździejewska [2], with optimal number of meals declared by $68.5 \%$ of young swimmers. Different findings were presented by Leonkiewicz et al., who assessed the number and timing of meals in a group of adolescent athletes, with regular consumption of 4-5 meals daily declared by only $20 \%$ of respondents [20] .Catyniuk et al., who assessed health-related and eating behaviours among students attending sport classes, showed that most respondents had more than 5 meals daily $(60.2 \%)$ as compared to only $6.6 \%$ of adolescents in our study [4].

Our study showed that $96.5 \%$ of dancers have snacks in between meals, with $63.6 \%$ of respondents opting for fruit and vegetables. Bielec and Goździejewska obtained similar results for vegetables and fruits as the most preferred snacks [2]. By contrast, Catyniuk et al. found that only $29.8 \%$ of respondents declared consumption of fruit, and that sweets were the most popular snack (68.8\%) [4].

In our study, the frequency of consuming different meals varied. Breakfast and supper were consumed by $87.4 \%$ and $80.3 \%$ of dancers, respectively. Similar findings for breakfast and different findings for supper, which was consumed by only $54 \%$ of respondents, were reported by Bielec and Goździejewska [2]. Different frequency of breakfast consumption was observed by Manore et al [24], according to which $55.7 \%$ of the participating footballers declared their daily consumption. On the other hand, in the study by Lim et al. [21] showing the lifestyle of Korean teenage women, $31.1 \%$ of the examined girls did not eat their first breakfast.

Since vegetables and fruits should form the basis of a balanced diet, their daily consumption is recommended. These products provide many valuable ingredients such as vitamins, antioxidants, minerals and fibre [17]. They significantly reduce the incidence of cardiovascular diseases and some types of cancer [35]. For these reasons, it is recommended that fruit and vegetables account for at least half of the food intake, with the proportion of $3 / 4$ of vegetables and $1 / 4$ of fruit [37].

Our study showed that $49 \%$ of dancers consumed fruit several times daily, with higher consumption rates among 10-12 year olds (51.3\%) than 13-15 year olds (45.6\%). Slightly higher frequency of fruit consumption was reported by Bielec and Goździejwska, with 58.6\% 
of respondents declaring consumption of fruit several times daily [2]. It may be seen from our study that $36.9 \%$ of dancers follow the recommendations to have several portions of vegetables per day. It should be also noted that consumption of vegetables several times daily was more common among younger than older respondents ( $42 \%$ vs. $29.1 \%)$. Similar findings were presented by Leonkiewicz et al. with $42.6 \%$ of students of the Athletic Championship School in Krakow declaring consumption of vegetables several times daily. Unfortunately, the study also identified a large group of children $(26.2 \%)$ who consumed vegetables less often than once daily [20]. A higher percentage (74\%) of people not consuming the recommended amount of vegetables was observed in their study by Coutinho et al [5]. Legumes are not an important part of the diet of the study group of dancers. Most of our respondents $(46.5 \%)$ consume these products several times a month. Similar findings were reported by Bielec and Goździejewska, with $56.2 \%$ of respondents declaring legume consumption less often than several times a week [2]. Different findings were presented by Coutinho et al. [5] in which $95 \%$ of athletes consumed legumes 5 or more times a week.

Bread is consumed several times a day by $57.1 \%$ of respondents. Similar results were obtained by Bielec and Goździejwska, with $50 \%$ of respondents consuming bread 2-3 times per day [2].

Yoghurts and kefirs are products with high nutritional values, providing the body with calcium, vitamin $\mathrm{D}$ and $\mathrm{A}$, as well as $\mathrm{B}$ vitamins, which is particularly important for active individuals [3]. Minerals contained in yoghurt, such as magnesium, calcium, phosphorus and potassium, can have a significant impact on bones [36]. Our study showed that $43 \%$ of dancers consume the above products several times a week, and that only $23.2 \%$ of respondents consume them on a daily basis. Similar observations were made by Calyniuk et al., with yoghurts and kefirs consumed a few times a week and every day by $39.8 \%$ and $40.9 \%$ of respondents [4].

Our study revealed insufficient intake of fish, which should be eaten 1-2 times a week [37]. Fish are an excellent source of not only easily digestible and absorbable protein, but also fats, vitamins, such as A, $\mathrm{D}$ and group $\mathrm{B}$ vitamins, and minerals, including $\mathrm{Ca}$, $\mathrm{P}, \mathrm{Fe}$ or Mg. Additionally, fish are an excellent source of iodine. They also contain omega-3 polyunsaturated fatty acids needed for the proper functioning of the brain, nervous system and eyesight, as well as for preventing heart diseases $[13,25]$. Our study showed that $55 \%$ of adolescents consume fish several times a month. Bielec and Goździejewska also observed poor consumption of fish, with $52.1 \%$ of respondents consuming fish less often than 2-3 times a week [2]. Catyniuk et al. showed that more than half of respondents consume fish 1-2 times a week, and 32.3\% of respondents eat fish occasionally [4].

Meat and lunch meats are another important group of products that should be included in the daily menu. These products are most often consumed several times a week by $44.9 \%$ of our adolescent respondents. Catyniuk et al. also showed that $52.7 \%$ of adolescents consume lunch meats several times weekly, including $37.6 \%$ of respondents consuming poultry 3-4 times a week [4].

Sweets are characterised by a low nutritional value, but a high content of sugars, fats, dyes and flavours [23]. Furthermore, eating sweets often leads to weight gain, as well as increased blood glucose and triglycerides [15]. The frequency of consumption of sweets was alarming in our study as $96.5 \%$ of all dancers declared consumption of these products, including 35.9\% of respondents eating sweets several times a week. Similar findings were reported by Dolipska et al. [7], who assessed eating behaviours among primary school students. As demonstrated by the authors, consumption of sweets several times a week was declared by $32.64 \%$ out of 573 girls and $30.62 \%$ out of 565 boys. Slightly lower frequency of consuming sweets was reported by Bielec and Goździejewska [2].

The questionnaire also asked about the frequency of consuming fast-foods. We found that $66.7 \%$ of dancers consume these products several times a month. Similar results were obtained by Tawfik et al. [33], who assessed eating patterns and supplement use among Egyptian athletes. According to the authors of the study, 57.8\% of the participants declared consumption of fast food once a week [33]. By contrast, Catyniuk et al. [4] found that $64.5 \%$ of children and adolescents consumed fastfoods occasionally, and Szczepańska et al. [32] showed that $52.96 \%$ out of 304 students also consumed these products on occasional basis. Eating fast foods leads to gastrointestinal disorders through insufficient supply of dietary fibre, which has a negative impact on health [30]. Furthermore, deep-fried foods are often a source of trans fats. They have a negative impact on health, particularly on an increased risk of cardiovascular diseases that lead to metabolic syndrome [6].

Individuals with increased physical activity require reliable nutrition education due to their increased demand for nutrients, as confirmed by the above cited studies. It is recommended that this education is also addressed to their closest environment to increase their nutritional awareness $[2,4,17]$. An important aspect seems to be conducting further research in order to identify the differences and the resulting nutritional needs in individual groups, taking into account: dance style, training intensity and exercise season. 


\section{CONCLUSIONS}

Eating behaviours and the frequency of consumption of food products by young dancers can be described as varied. Their diet is characterised by regular consumption of meals and a correct frequency of consumption of most food products, including high intake of fruit and vegetables, yoghurt and kefir, as well as low consumption of fast food products.

The frequency of consumption of second breakfast, afternoon snack, and sweets significantly increases with age, while the frequency of fruit consumption significantly decreases.

The selected elements of dancers' lifestyle, such as sleep time and physical activity, are mostly correct.

\section{Conflict of interest}

The authors declare no conflict of interest.

\section{REFERENCES}

1. Ali A., Al-Siyabi M.S., Waly M.I., Kilani H.A.: Assessment of Nutritional Knowledge, Dietary Habits and Nutrient Intake of University Student Athletes. Pakistan Journal of Nutrition 2015;14 (5): 293-299.

2. Bielec G., Goździejewska A.: Nawyki żywieniowe 11-12-letnich uczniów sportowych klas pływackich na tle nietrenujących rówieśników - badanie pilotażowe [Nutritional habits of 11-12-year-old swimmers against non-athlete peers - a pilot study]. Pediatr Endocrinol Diabetes Metab 2018;24,2:72-81(in Polish).

3. Broad EM., Cox GR.: What is the optimal composition of an athlete's diet? Eur J Sport Sci. 2008;8(2):57-65.

4. Catyniuk B., Kiciak A., Grochowska-Niedworok E.: Zachowania zdrowotne i żywieniowe uczniów szkół o profilu sportowym ( $\mathrm{z}$ uwzględnieniem płci oraz wskaźnika BMI badanych) doniesienie wstępne [Healthand nutrition-related behaviour of pupils attending sports schools in relation to their gender and body mass index - preliminary report]. Medycyna Ogólna i Nauki o Zdrowiu, 2014;20(2),186-191 (in Polish).

5. Coutinho A., Porto C., Pierucci A.: Critical evaluation of food intake and energy balance in young modern pentathlon athletes: a cross-sectional study. Journal of the International Society of Sports Nutrition. 2016 13:15.

6. Dąbkowska M.: Wpływ spożycia kwasów tłuszczowych trans TFA na rozwój zespołu metabolicznego [Effect of consumption of trans tfa fatty acids on the development of the metabolic band]. "Acta Salutem Scientiae" 2019,1:41-51 (in Polish).

7. Dolipska A., Góra A., Janion K., Szczepańska E.: Zachowania żywieniowe dziewcząt i chłopców uczęszczających do wybranych szkół podstawowych [Dietary patterns of primary school pupils according to their sex]. Pom.J.Life Sci. 2019:65(3),104-109 (in Polish).
8. Dymarska E., Grochowalska A., Jaskułka - Błaszak M., Porażka J., Krauss H., Chęcińska Maciejewska Z.: Wpływ sposobu odżywiania na układ odpornościowy w różnych grupach wiekowych [Influence of nutrition on immune system in different age groups]. Probl Hig Epidemiol 2017; 98(1):63-72 (in Polish).

9. Freeman C., Zehra A., Ramirez V., Wiers C., Volkow $N$., Wang G.: Impact of sugar on the body, brain, and behavior. Frontiers In Bioscience 2018;23;2255-2266.

10. Ginter E., Simko V.: New data on harmful effects of trans-fatty acids. Bratisl Lek Listy. 2016;117(5):251-3.

11. Jarosz M.: Normy żywienia dla populacji Polski [Nutrition standards for the Polish population]. Instytut Żywności i Żywienia, Warszawa 2017.

12.Jarosz M., Charzewska J., Wajszczyk B., Chwojnowska $Z$ :: Czy wiesz, ile potrzebujesz białka? [Do you know how much protein you need?]. Warszawa, Instytut Żywności i Żywienia, 2019.

13. Kaliniak A.: Wpływ sezonu pozyskania wybranych gatunków ryb z polskiej akwakultury na profil kwasów tłuszczowych i wskaźniki żywieniowe lipidów ich mięsa [Effect of fishing season of selected fishes farmed in poland on meat fatty acid profile and dietary indices of lipids in their meat]. Żywność. Nauka. Technologia. Jakość 2019 ;26(2):70-82 (in Polish).

14. Kalisz Z., Juraszek K., Glama E., Weber-Rajek M., Hoffmann M., Kalisz J., Zukow W.: Składniki odżywcze $\mathrm{i}$ ich rola w diecie sportowca [Nutrients and their role in athlete's diet]. Journal of Education, Health and Sport. 2016;6(8):522-538 (in Polish).

15. Kaniewska E., Gaździńska A., Jagielski P., Gaździński S., Wyleżot M.: Ocena częstotliwości spożycia wybranych produktów spożywczych przez chorych zakwalifikowanych do zabiegowego leczenia otyłości [Assessment of selected food intake frequency in obese patients qualified for bariatric surgery]. Hygeia Public Health 2016,51(1): 66-70 (in Polish).

16. Kasek M.: Zmiany w zwyczajach żywieniowych Polaków [Changes in dietary habits of Polish people]. Przemysł Spożywczy 2016;70(12):2-7 (in Polish).

17. Kucharska A., Oleksiak N., Sińska B., Zegan M., Michota-Katulska E.: Warzywa i owoce źródłem witamin i składników mineralnych w diecie studentek dietetyki [Fruits and vegetables as a source of vitamins and minerals in the diet of female students of dietetics]. Bromat Chem Toksykol 2016,2:145-151 (in Polish).

18. Kwiatkowska M., Wawrzyniak A.: Edukacja żywieniowa jako element poprawiający zachowania żywieniowe oraz wybrane parametry antropometryczne w grupie dzieci w wieku 12 lat [Nutritional education as an element improving nutritional behavior and selected anthropometric parameters in the group of children aged 12 years] Piel Zdr Publ. 2019;9(3):173-182 (in Polish).

19.Laskowski W., Górska-Warsewicz H., Rejman K., Czeczotko M., Zwolińska J.: How Important are Cereals and Cereal Products in the Average Polish Diet? Nutrients. 2019;11(3):679.

20.Leonkiewicz M., Gacek M., Frączek B.: Wiedza i zachowania żywieniowe młodzieży uprawiającej sport 
- konieczność edukacji [Knowledge and nutritional behavior of adolescents practicing sport - the need for education].; red. A. Wolska-Adamczyk, WSIiZ, Warszawa 2015 (in Polish).

21. Lim H.S., Kim T.H., Lee H.H., Park Y.H., Lee B.R., Park Y.J., Kim Y.S.: Fast food consumption alongside socioeconomic status, stress, exercise, and sleep duration are associated with menstrual irregularities in Korean adolescents: Korea National Health and Nutrition Examination Survey 2009-2013. Asia Pac J Clin Nutr. 2018;27(5):1146-1154.

22.Łopuszańska-Dawid M.: Jaki powinien być poziom aktywności fizycznej dzieci i młodzieży szkolnej? [What should be the level of physical activity of school children and youth?] Available https://ncez.pl/ aktywnosc-fizyczna/dzieci-i-mlodziez/jaki-powinienbyc- poziom-aktywnosci-fizycznej-dzieci-i-mlodziezyszkolnej- (Accessed: 04.06.2020).

23.Malczyk E., Zołoteńka-Synowiec M., Całyniuk B., Malczyk A., Synowiec J.: Częstotliwość spożycia wybranych produktów spożywczych przez studentów opolskich, śląskich i dolnośląskich uczelni [The frequency of consumption of selected food products by students from Opole Voivodship, Lower Silesia and Silesian universities]. Piel Zdr Publ. 2017;26(1):35-43 (in Polish).

24.Manore M.M., Patton-Lopez M.M., Meng Y., Wong S.: Sport Nutrition Knowledge, Behaviors and Beliefs of High School Soccer Players. Nutrients 2017, 9, 350.

25. Marciniak-Eukasiak K.: Rola i Znaczenie Kwasów Tłuszczowych Omega-3. [The role and significance of omega 3 fatty acids]. Żywność Nauka Technologia Jakość 2011,6(79):24-35 (in Polish).

26. Nascimento M., Silva D., Ribeiro S., Nunes M., Almeida M., Mendes-Netto R.: Effect of a Nutritional Intervention in Athlete's Body Composition, Eating Behaviour and Nutritional Knowledge: A Comparison between Adults and Adolescents.Nutrients 2016, 8, 535.

27. Obrenovich M., Siddiqui B., McCloskey B., Reddy V.P.: The Microbiota-Gut-Brain Axis Heart Shunt Part I: The French Paradox, Heart Disease and the Microbiota. Microorganisms. 2020;8(4):490 doi:10.3390/ microorganisms8040490.

28.Rosa D.D., Dias M.M.S., Grześkowiak L.M., Reis S.A., Conceição L.L., Peluzio M.D.C.G.: Milk kefir: nutritional, microbiological and health benefits. Nutr Res Rev. 2017;30(1):82-96.

29. Saini R.K., Keum Y.S.: Omega-3 and omega-6 polyunsaturated fatty acids: Dietary sources, metabolism, and significance - A review. Life Sciences 2018;15;203:255-267.
30.Shau J.P., Chen P.H., Chan C.F., Hsu Y.C., Wu T.C., James F.E., Pan W.H.: Fast foods-are they a risk factor for functional gastrointestinal disorders? Asia Pac J Clin Nutr 2016;25(2):393-401.

31. Szczebyło A., Halicka E., Łuczyńska K.: Spożycie nasion strączkowych w Polsce jako element modelu zróżnicowanej konsumpcji żywności [Consumption of pulses in Poland as an element of a sustainable food consumption model]. Prace Naukowe Akademii im. Jana Długosza w Częstochowie, Pragmata tes Oikonomias 2018,12:35-45 (in Polish).

32. Szczepańska E., Janota B., Janion K.: Selected lifestyle elements in adolescents attending high schools. Rocz Panstw Zakl Hig. 2020:71(2):147-156, doi: 10.32394/ rpzh.2020.0109

33. Tawfik S., Koofy N., Moawad E.: Patterns of Nutrition and Dietary Supplements Use in Young Egyptian Athletes: A Community-Based Cross-Sectional Survey. PLOS ONE. 2016:11(8).

34. WHO. Global recommendations on physical activity for health. Geneva 2010. Available https://apps.who.int/iris/ bitstream/handle/10665/44399/9789241599979_eng. pdf?sequence $=1$ (Accessed 30.06.2020).

35. Wolnicka K., Jaczewska-Schuetz J., Taraszewska A., Charzewska J., Jarosz M.: Częstotliwość spożycia warzyw i owoców przez dzieci i młodzież w szkołach podstawowych i gimnazjach w Polsce [Requency of consumption of vegetables and fruits by children and adolescents in primary and lower secondary schools in Poland]. Żywienie Człowieka i Metabolizm 2017;44(4):258-267 (in Polish).

36. Wyka J., Tajner-Czopek A., Rytel E., Habanova M., Malczyk E., Misiarz M.: Żywność fermentowana znaczenie dla zdrowia człowieka [Fermented food meaning to human health]. Acta Sci. Pol. Biotechnologia 16(1-4)2017:101-106 (in Polish).

37. Zasady prawidłowego żywienia - Piramida Zdrowego Żywienia i Aktywności Fizycznej [Principles of healthy eating - Pyramid of Healthy Nutrition and Physical Activity]. Available http://www.izz.waw.pl/zasadyprawidowego-ywienia (Accessed: 22.06.2020).

38. Zerek U: Sztuka i Dokumentacja Taniec jako sztuka relacji [Art and Documentation. Dance as the Art of Relationships]. 2016;14:118-143 (in Polish).

Received: 30.11 .2020

Accepted: 23.02.2021 